\title{
A Common Vision:
}

A Strategy for Integrated Vegetation Management

In the Bureau of Land Management

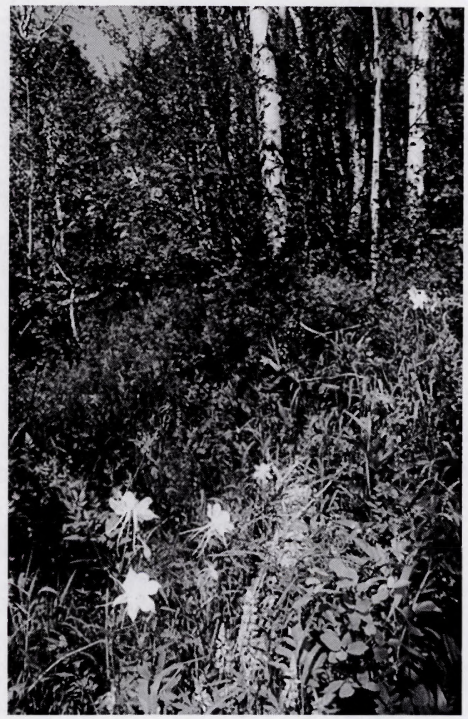

.v43 


\section{Table of Contents}

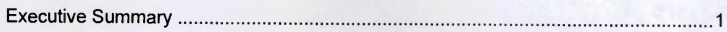

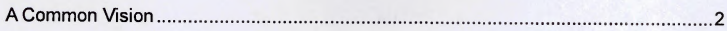

Eating the Elephant a Bite at a Time .......................................................................

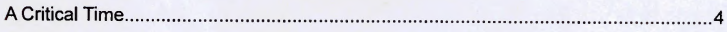

What is an Integrated Vegetation Management Strategy? ........................................4

How This Strategy Fits With the BLM and DOI Strategic Plans .................................5

The Relationship between This Strategy and the BLM Vegetation

Treatments EIS and Programmatic Environmental Report ......................................5

The Relationship Between this Strategy and Other DOI and BLM Initiatives..............6

Your Help is Needed to Develop this Strategy .....................................................6

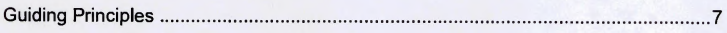

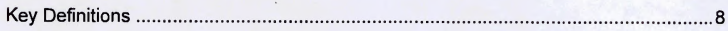

Pulling It Together: Six Key Vegetation Management Issues ..................................... 9

Issue 1: Align Program Objectives and Priorities ......................................... 9

Issue 2: Develop Complementary Assessment Approaches.............................10

Issue 3: Align Land-use Planning Direction................................................10

Issue 4: Integrate Our Program of Work ..........................................................11

Issue 5: Improve Management Practices …..............................................11

Issue 6: Align Monitoring Procedures and Direction ...................................12

BLM Library

Denver Federal Center

Bldg. 50, OC-521

P.O. Box 25047 
Yas7dif M_L8 7otre rsa-00, oè pble THOCS xog 0.9 aSS08 00.19vrä 


\section{Executive Summary}

"It will be the century of ecological restoration or it will be nothing at all."

Stephen Ambrose

Vegetation is perhaps the one uniting feature of all the 261 million acres cared for by the Bureau of Land Management (BLM). Yet history shows how quickly this core resource can be changed by invasive weeds, wildfire, drought, grazing, recreational use and other activities.

BLM recognizes the importance of vegetation management. That recognition is symbolized in the 150-plus different authorities or guidelines that exist for vegetation management within the agency. But that huge number also illustrates the central problem: A common approach for managing vegetation, one that can be embraced by a variety of disciplines across the agency, is lacking. As it stands now, BLM's approach to vegetation management is often fractured, with insufficient crossover among resources and programs to achieve a cohesive, coordinated effort.

BLM cannot afford to approach vegetation management in a disjointed manner. Too much is at stake. Now is possibly the best chance of developing and putting into practice a strategy that will help us to achieve sustainable healthy landscapes across public land in the West.

In June 2005, the BLM Director tasked a multi-disciplinary team to develop an integrated vegetation management strategy to align vegetation management policies, priorities and direction within BLM. The team has identified six specific areas needing attention: Align program objectives and priorities; develop a common approach to describing vegetation condition; align land-use planning direction; remove barriers to help integrate a program of work; improve management practices; and, align monitoring procedures and direction. This common vision is the first step in accomplishing the Director's objectives. 


\section{A Common Vision}

"The outstanding scientific discovery of the twentieth century is not television or radio, but rather the complexity of the land organism. Only those who know the most about it can appreciate how little we know about it."

Aldo Leopold

The one uniting feature of the 261 million acres of land under the Bureau of Land Management's (BLM) care is the plants - grasses, forbs, shrubs, and trees - that grow upon it. Allow the vegetation resource to deteriorate and the land itself loses its value, declining as habitat, lacking in visual and recreational appeal, depriving it of its ability to sustain communities, and bringing to an end the chain of goods, services and opportunities that exist primarily because of the vegetation the land supports. Recent history shows vegetation's ability to change dramatically for the worse. In the Great Basin alone, more than 25 million acres of public land is now dominated by invasive, non-native species, causing a huge drop in productivity and resource health. A significant portion of what BLM does as an agency is tied directly to what grows on the land. It's that simple and that complex. As Aldo Leopold wrote, only those who know the most about the land understand how little we really understand.

Recognition of that fact makes the management of vegetation one of the most vital and crucial of BLM's responsibilities. It shows in the vast number of policy, guidance, planning, legislative and general principles that the agency deals with when it comes to managing vegetation. Further, an array of resource disciplines, from rangeland to forestry to fire to weed management and beyond, has its own take and methods of how vegetation management should be completed. A survey of current vegetation management and policy guidance reveals more than 150 different authorities or guidelines for vegetation management within the agency. Sometimes, these efforts are complementary. At other times, they are at odds with one another. Some are well known, some are collecting dust on bookshelves and in desk drawers.

All of this points to one clear fact. Given the importance of the vegetation resource and the multitude of guidance, it's imperative that BLM pull together a common approach for restoring land health through integrated vegetation management. It's important, too, that policies and practices at odds with each other be identified and reconciled. It's critical, to the degree possible, that the disciplines' approaches to vegetation management be aligned under one common vision, pointed toward one common goal. 

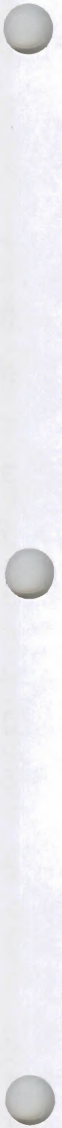


\section{Eating the Elephant a Bite at a Time}

Several attempts have been made to produce that kind of alignment, but for a variety of reasons, not much has materialized. In June 2005, a multi-resource team met in Boise, Idaho, to begin the work of aligning vegetation management policies, priorities and direction within BLM. One of the main goals of the team was to help the field develop integrated work plans and meet program land-health goals more efficiently. A specific assignment was to reconcile the land health assessment processes being used in different program areas and develop common objectives and priorities for restoring land health, while coordinating efforts with the Bureau's Monitoring Strategy Team, and considering the work's relationship with the Vegetation Treatments on BLM Lands Environmental Impact Statement, the Great Basin Restoration Initiative, and other related efforts. The team also recognized that each program contains unique vegetation management goals and objectives, which should be accounted for in whatever guidance is eventually produced.

The team assignment was ambitious. Several subgroups were organized to address broad topics. The topics examined by the subgroups are:

1. Align program objectives and priorities and improve resource allocation to meet common vegetation management goals.

2. Develop a common approach to describing and assessing vegetative condition.

3. Align land-use planning direction for vegetation management in land-use planning.

4. Design a process for removing barriers to help integrate a program of work.

5. Improve management practices to foster land-health objectives.

6. Align monitoring procedures and direction, including coordinating efforts with the National Monitoring Strategy Team and with the Wildland Fire Leadership Council's monitoring strategy.

In a subsequent meeting in Boise in February 2006, the subgroups began work on an integrated vegetation management strategy, including recommendations. This report represents a summary of their work.

This report is divided into two major parts. The first is the narrative, which gives an overview of the vegetation management efforts and what the team thinks should be done to resolve issues. The second part of the report is the "Action Plan," which takes the form of a statement of the current situation and actions recommended by the team to resolve the issue. Together, the narrative and the Action Plan provide a general guide for what should take place regarding integrated vegetation management in BLM. The ultimate product of the vegetation management team and the work of others will be a handbook that incorporates the information and direction needed by field offices throughout BLM. 

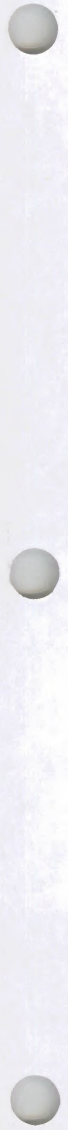


\section{A Critical Time}

The ultimate goal of the team is to provide guidance that will ensure a uniform approach to vegetation management that will improve the health and productivity of BLM land, while still recognizing that some needs are unique to each discipline. Right now, BLM is at a critical point. Threats to public land may never have been more substantial - weeds, wildfire, drought, and pressure from a society demanding open space, recreational opportunities, grazing use, timber products and energy production, all coming at a time when BLM is facing a period of declining budgets. Healthy, productive land has never been at more of a premium. A coordinated plan for the vegetation that it supports has also never been needed more than now.

\section{What is an Integrated Vegetation Management Strategy?}

The dictionary defines a "strategy" as a plan for achieving an identified goal. It also defines the term "integrate" to mean uniting different components toward a common purpose. In the case of an integrated vegetation management strategy, our aim is to unite the various programs within BLM toward achieving a common goal of protecting, maintaining and restoring ecologically diverse and properly functioning native plant communities on public land.

BLM's resource programs evolved from legal requirements that are frequently focused on specific resource uses and requirements. As such, policies and procedures are often developed that are unique to a program area, sometimes making it difficult to focus on a common purpose. One example is the unique protocols for assessing range, fire, and wildlife habitat conditions.

These various program policies and procedures can lead to different program priorities and can confuse the public about our overall goal for vegetation. After all, these programs rely on the same vegetative resources to achieve their individual program goals. It makes sense to work together to achieve a common outcome for vegetation, one that will support all of the renewable resource programs.

This strategy will capitalize on the strengths and capabilities of each of the renewable resource programs to gain efficiency toward what has become over the years a common goal: To protect, maintain and, where necessary, restore the ecological diversity and resiliency of native plant communities on public land.

The strategy acknowledges the problems faced by public land managers are bigger than any one program can successfully tackle. Managers face formidable challenges, including invasive, alien plants (such as cheatgrass, red bromegrass, yellow starthistle, and leafy spurge); altered fire regimes; and the long-lasting effects of drought and the potential management implications associated with climate change. All of these can mean potential alteration or devastation to plant communities and ecosystems. 

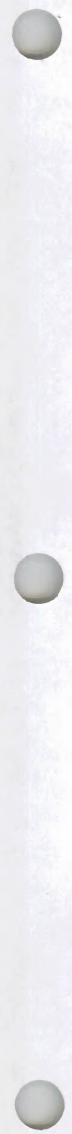
We need to work together toward a common vision of more resilient and productive native plant communities on public land if we are to be successful in resolving these problems.

Finally, BLM needs to send a strong, unified message to the public and our stakeholders: We have a shared commitment to improve land health and provide sustainable public land uses for our children, grandchildren and generations beyond.

\section{How This Strategy Fits With the BLM and DOI Strategic Plans}

Resource protection is a cornerstone of both the Department of the Interior (DOI) and BLM Strategic Plans. Both recognize that multiple use and sustained-yield principles are best served by healthy and productive land, including the vegetation. By focusing all of our programs on protecting, maintaining and restoring native plant communities on public land, we can be more successful at fulfilling a vital part of those strategies.

\section{The Relationship between this Strategy and the BLM Vegetation Treatments EIS and Programmatic Environmental Report}

As stated in the draft BLM Vegetation Treatments Environmental Impact Statement (EIS) and Programmatic Environmental Report (PER), released for public comment in November 2005, vegetation management on public land is a vital function of the agency. The draft EIS/PER analyzed a potentially significant increase in the amount of vegetation that could be treated to respond to Presidential and Congressional mandates to reduce the risk of wildfire, restore fire-adapted ecosystems and repair land damaged by fire.

The draft Vegetation Treatments EIS/PER provides the rationale for such an increase and describes the types of treatments that can be used, best management practices to be applied, and discloses the potential environmental effects of such a program. When final, it will provide documentation to which BLM field offices can tier and/or reference in making decisions to implement vegetative treatments.

This strategy is designed to guide implementation of the program of work outlined in the Vegetation Treatments PER through a coordinated and integrated approach. 

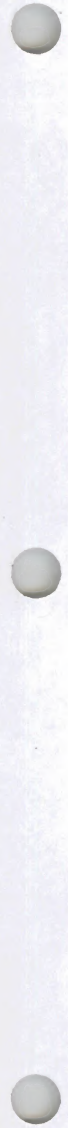


\section{The Relationship Between this Strategy and Other DOI and BLM Initiatives}

This strategy capitalizes on a common thread running through many other vegetation management initiatives that affect BLM, all of which emphasize that healthy and properly functioning native plant communities provide the most sustainable and lowest-risk condition to achieve their particular goals and objectives. Even though these initiatives may have been triggered by a specific event or issue, they all retain a common component of managing vegetation to achieve a particular purpose.

These initiatives include:

- Great Basin Restoration Initiative

- National Fire Plan

- Ten-Year Comprehensive Strategy Implementation Plan

- Cohesive Strategy -- Protecting People and Natural Resources

- Healthy Forests Initiative

- Healthy Forests Restoration Act

- Sage Grouse Strategy

Building upon the work already completed in these initiatives, and combining those efforts into an overarching vegetation management strategy, BLM will be in a better position to achieve consistent, coordinated, and integrated vegetation management goals.

\section{Your Help is Needed to Develop this Strategy}

The initial team of resource experts and program representatives asked to develop a vegetation management strategy included representatives from all of the renewable resource programs (range, forestry, weeds, wildlife, recreation, cultural and heritage resources), fire programs (fuels and fire planning), planning, National Land Conservation System (NLCS), and the minerals, realty, and resource protection group, as well as The Nature Conservancy.

The Vegetation Management Strategy Team was charged with developing recommendations for actions that BLM could take within the current legal and regulatory authorities that would unite all programs and initiatives associated with vegetation management. In short, a common approach with a common purpose was needed. Much progress has been made in developing the strategy. Now, it's time to get broader involvement in developing and implementing this strategy.

That's where your help is needed. We are reaching out to our field offices, the public, public land stakeholders, and other interest groups to help us refine this strategy. This document has been published to provide consistent information about the intent and progress of this strategy to date. Please take a look at it, and let us know your thoughts on how we can improve vegetation management on public land. You may send your comments by e-mail to Rick Tholen (rick_tholen@blm.gov) or Roy Johnson (roy_johnson@blm.gov) the co-leaders of the vegetation management strategy team. 

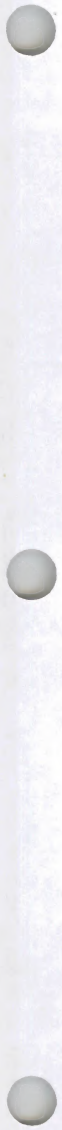


\section{Guiding Principles}

The following principles will be used to guide further development of this strategy and an implementation action plan.

- Diverse, healthy, and resilient native plant communities provide the greatest opportunity to be successful in meeting objectives for all programs within BLM.

- Plant communities integrate at a landscape scale; they react to biological and physical conditions and disturbances that play out over large areas. Therefore, the goal should not be to have native plant communities on every acre of public land. Rather, the goal is to manage a mix of plant communities comprised primarily of native species across the landscape, thus allowing for a number of uses to co-exist with these broader landscape plant communities.

- Decisions concerning the desired mix of plant communities and uses will be made at the local level, through the land-use planning and implementation process, with involvement of local communities, stakeholders, other landowners, tribes and other agencies. This approach will help avoid duplication of efforts, ensure consistency and improve public acceptance of vegetation management activities.

- Renewable resource programs and processes within BLM should be structured to promote working toward common goals and objectives that will maximize the effectiveness of our management actions, as well as improve overall program efficiency. This can be accomplished through a collaborative, interdisciplinary process, based on the best available science.

- The Land Health Standards developed in conjunction with local Resource Advisory Councils will serve as a framework within which vegetation condition will be assessed and described. BLM has made significant progress in defining and gaining consensus on land health issues using the Land Health Standards process. This effort will seek to build upon this progress.

- Programs will continue to have specific objectives, such as use objectives, that will be consistent with the overarching shared goals and objectives described in this strategy.

- An integrated, interdisciplinary approach in planning, implementing and monitoring management actions, regardless of which program is funding the work, will improve efficiency and help achieve our multiple-use and sustained-yield mission.

- An interdisciplinary approach to integrated vegetation management will be established at all levels of BLM, from the national office to field offices. 

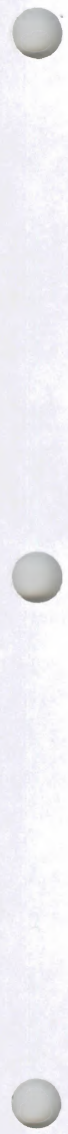


\section{Key Definitions}

\section{Desired Plant Community}

The kind, proportion and amount of vegetation necessary for achieving the objectives established for an area

\section{Diverse Plant Community}

A large assemblage of plants with adequate representation from all the species groups that would be found in a nondegraded environment of that type.

\section{Healthy Landscape}

A healthy landscape is one where plant communities, wildlife and other living creatures and ecological processes (including natural fire regimes) are functioning appropriately and on a biologically sustainable basis. Appropriate uses and activities can be supported on healthy land now and into the future.

\section{Invasive Plants}

Plants that are not part of (if exotic) or a minor component of (if native) the original plant community or communities that have the potential to become a dominate or co-dominant species on the site if their future establishment and growth is not actively controlled by management interventions, or are classified as exotic or noxious plants under state or federal law. Species that become dominate for only one to several years (e.g. short-term response to drought or wildlife) are not invasive plants.

\section{Landscape}

A mosaic of several plant communities, usually that portion of land that the eye can comprehend in a single view, including all natural characteristics. The area can range from a couple of hundred acres to tens of thousands of acres.

\section{Native Plant Communities}

Plant communities having the proper mix of native species, structures, and landscape mosaic consistent with the natural disturbance regime.

\section{Protection}

Taking action to prevent further degradation of a native plant community, such as through construction of fire breaks, control of weeds and non-native invasive species or altering uses.

\section{Renewable Resource Programs}

BLM programs that have as part of their management focus the biological resources that occupy the public land, including forestry, rangeland, wildlife, weeds, botany, recreation and hazardous fuels management.

\section{Resilient Plant Community}

A plant community with the ability or capacity to renew its composition, structure and function following disturbance.

\section{Restoration}

Implementing a series of actions that promote plant community diversity and structure that allows them to be more resilient to disturbance and the threat of invasive exotic species over the long term.

\section{Vegetation Management}

Purposeful actions that are a result of an assessment of vegetation conditions and that meet land-use plan objectives. 
$$
0
$$
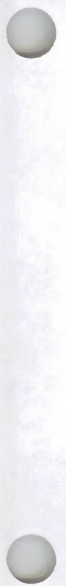


\section{Pulling It Together: Six Key Vegetation Management Issues}

"A root, a stem, a leaf, some means of capturing sunlight and air and making food - in sum, a plant. The green substance of this earth, the chlorophyll, is all summed up in the plants. Without them, we perish, all of us who are flesh and blood."

\section{Hal Borland}

Six key issues were identified and addressed by the Vegetation Management Strategy Team. The summary of these issues and the team's recommendations follows.

\section{Issue 1: Align Program Objectives and Priorities}

\section{Current Situation:}

The practice of independently developing program-specific policy, practices and procedures does not facilitate an integrated approach to managing vegetation on public land. Multiple programs in BLM address vegetation management, but their approach and desired outcomes are not always consistent. This can reduce the overall effectiveness of BLM's efforts to restore and maintain land health and provide sustainable use.

\section{Recommended Actions:}

- Develop a common vegetation management goal for all programs. Focus this common goal on restoring or maintaining diverse and resilient native plant communities. Diverse and resilient native plant communities that provide the greatest opportunity for success. Resilient native plant communities respond to disturbance in a predictable way and recover after the disturbance and resist being taken over by invasive plant species.

- Evaluate existing BLM and other agency policies, handbooks, practices and procedures and identify conflicting policies and procedures in order to develop and provide consistent direction to the field. Develop a matrix that identifies both common and conflicting policies.

- Develop common restoration priorities across all programs as appropriate.

- Identify common vegetation terminology to be used throughout the BLM.

- Develop an integrated vegetation management handbook to guide vegetation management for all renewable resource programs.

- Establish a standing vegetation management oversight group with representation from all renewable resource, fire, minerals, and National Landscape Conservation System programs to address issues that arise during implementation of this strategy. 
.
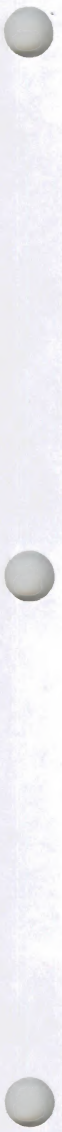


\section{Issue 2: Develop Complementary Assessment Approaches}

\section{Current Situation:}

Different programs have different approaches to assess and manage vegetation, based on the particular focus of each program. While these different approaches are not necessarily in conflict, nor are they always complementary or facilitate a larger, integrated approach. For example, an emphasis on single-species focused assessments can increase the effort needed to later integrate vegetation/habitat assessments. Another example is where rangeland health is assessed using ecological site descriptions by the range program and the fire program assesses the same area and comes to a different conclusion using other protocols.

\section{Recommended Actions:}

- Analyze similarities and differences among the reference conditions and the use of scales of different assessment methods. Investigate the potential to integrate assessment methods.

- Develop a case study that describes how to integrate results from the different assessment approaches that would provide a sample or template for field offices to use.

- Provide direction for using integrated assessments to set goals in land-use plans and activity plans and to prioritize and select projects.

- Promote the integration of assessments, data collection and data storage throughout BLM.

- Identify indicators and procedures to be used in Land Health Assessments (LHA) to better describe the diversity and resiliency of vegetation on the landscape, as opposed to the allotment or management unit level.

\section{Issue 3: Align Land-use Planning Direction}

\section{Current Situation:}

The BLM Land-Use Planning Handbook (H-1601) establishes direction for implementing land-use planning requirements, including incorporating land health standards, developing desired future condition goals, and establishing conservation and restoration priorities within land-use plans. A number of program-specific requirements contained in $\mathrm{H}-1601$ could also foster program-specific goals and priorities within land-use plans. Furthermore, goals and objectives in land-use plans are not described at the same scale, and do not use the same naming conventions as in LHA, FRCC, Ecological Site Inventory, and other program-specific assessments. Likewise, goals and objectives are often not described in sufficient detail or use a uniform nomenclature to understand program-specific effects.

\section{Recommended Actions:}

- Provide clarification on how land health standards can be used to establish land-use plan goals and land health indicators can be used to establish multi-program objectives and priorities.

- Provide examples of integrated land use plan-level goals and priorities for vegetation management.

- Improve linkages between direction contained in Appendix C/Vegetation and other sections of Appendix $\mathrm{C}$ that affect vegetation. 

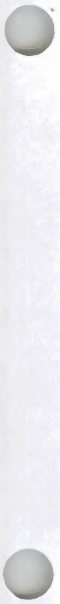

0 


\section{Issue 4: Integrate Our Program of Work}

\section{Current Situation:}

Work priorities are not always established in an integrated manner where diverse interests and skills and multiple funding sources can be brought to bear in addressing resource conditions. Several barriers exist to developing a more integrated program of work in the field, including different budget formulation requirements, programmatic budget allocation, different budget timeframes, different reporting requirements, and length of time integration requires.

\section{Recommended Actions:}

- Develop a consistent budget formulation process for all of the vegetation management programs, including development of a five-year program of vegetation management work for the field office level (including NEPA requirements, clearances, survey and design, coordination and consultation, contracting and permitting) that is used by the programs to determine preliminary and annual funding allocations. This program of work should reflect local, state, regional, and national priorities.

- Develop consistent annual work plan direction for vegetation management, including consistent objectives and priorities where possible.

- Develop a process to work together at the national level to address cross-program funding needs and priorities.

- Develop specific program elements for vegetation restoration to be used by all programs.

- Establish a single data base for project submission, funding, and accomplishment tracking that all vegetation management programs can utilize.

- Conduct periodic integrated evaluations of all vegetation management activities.

\section{Issue 5: Improve Management Practices}

\section{Current Situation:}

Existing BLM program structures do not facilitate a cohesive approach regarding best management practices (BMPs) for vegetation management. Currently, BMPs exist but they have been developed program by program and are not integrated with one another. Further, at times they may even conflict with each other. BLM is one of many agencies and organizations that use BMPs. Beyond BLM, these "outside" BMPs and existing research may be useful to our integrated management approach.

In addition, many staff members are unfamiliar with the pest management and native plant material programs. Additional information should be provided to the field concerning these programs. 



\section{Recommended Actions:}

- Review existing BMPs relative to vegetation management activities, including those contained in the Draft Vegetation Treatments EIS/PER.

- Assess the effectiveness of current BMPs and recommend changes or additions needed to achieve land health objectives related to vegetation.

- Identify a common set of BMPs to be used within BLM vegetation management programs and activities.

- As BMPs are developed, BLM must ensure that they are integrated across BLM programs; that they are based on the best available science and are peer reviewed; and that they are designed to maintain or restore the diversity and resiliency of native plant communities.

- Provide information to the field on the BLM pest management and native plant materials programs.

\section{Issue 6: Align Monitoring Procedures and Direction}

\section{Current Situation:}

Monitoring is at the heart of telling us if our management actions are meeting objectives at either the local or landscape level. There are a number of different ways of measuring vegetation change and assessing if this change is having the desired effect. Different programs use different monitoring techniques and protocols, which is not conducive to understanding if integrated objectives are being achieved.

While a monitoring strategy is required in land-use plans, specific direction is lacking, providing no examples of how it should be done, or how it links with other activities. Little attention is paid to addressing the key current, potential and desired condition of the vegetation.

\section{Recommended Actions:}

- Develop a template for a vegetation monitoring protocol to be used by all programs that monitor the effectiveness of treatments to maintain or restore vegetation diversity and resiliency.

- Develop a template for a standard report on vegetation condition at the local level.

- Identify a means for storing vegetation monitoring data just once, but allowing for it to be used as often as needed.

- Develop recommendations regarding the frequency and extent of mapping BLM projects and disturbance events (e.g., insect infestations, wildland fires). 


\section{Vegetation Management Strategy Team Members}

Rick Tholen, co-team leader, Renewable Resources and Planning (WO-200)

Roy Johnson, co-team leader, Fire Planning and Fuels Management (FA-600)

Lynda Boody, Fire Planning and Fuels Management (FA-600)

Ed Brunson, The Nature Conservancy, Boise, Idaho

Erik Christiansen, Fire Planning and Fuels Management (FA-600)

Tom Dabbs, Budget (WO-880)

Susan Giannettino, Deputy State Director, Idaho State Office (ID-930)

Carsten Goff, Rangeland Resources (Landfire) (WO-220)

Jack Hamby, Rangeland Resources (WO-220)

Doug Havlina, Fire Planning and Fuels Management (FA-600)

Mark Hilliard, Fish, Wildlife and Plant Conservation (WO-230)

Mike "Sherm" Karl, Vegetation Inventory, and Monitoring, National Science and Technology Center

Scott Lambert, Resources and Science, Idaho State Office (ID-931)

Elroy Masters, Div. Natural Resource, Lands \& Planning, Nevada State Office (NV-930)

(Representing Planning, Assessment and Community Support, WO 210)

Linda Mazzu, Fire Planning and Fuels Management (FA-600)

Ted Milesnick, Fire Planning and Fuels Management (FA-600)

Gregg Nelson, Forest and Woodland Management (WO-270)

Peggy Olwell, Fish, Wildlife and Plant Conservation (WO-230)

Mike Pellant, Great Basin Restoration Initiative, Nevada State Office (NV-910)

Jim Perry, Fluid Minerals (WO-310)

Doug Powell, Rangeland Resources (WO-220)

Gina Ramos, Rangeland Resources (WO-220)

Rod Roudabush, National Landscape Conservation System (WO-170)

Don Smurthwaite, External Affairs, Montana State Office (MT-912)

Steve Tryon, Rangeland Resources (WO-220)

Kate Winthrop, Cultural and Fossil Resources and Tribal Coordination (WO-240) 

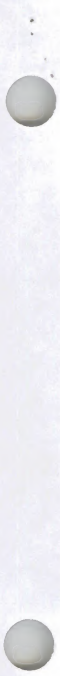